\title{
Characterisation of B cell epitopes of dengue virus NS1 protein using bioinformatics approach
}

\author{
P.D. Pushpakumara ${ }^{1,2}$, P.H. Premarathne $e^{1,2}$ and C.L. Goonasekara ${ }^{1,2^{*}}$ \\ ${ }^{I}$ Institute for Combinatorial Advanced Research and Education, General Sir John Kotelawala Defence University, Ratmalana. \\ ${ }^{2}$ Faculty of Medicine, General Sir John Kotelawala Defence University, Ratmalana.
}

\begin{abstract}
Linear B cell epitopes of the dengue virus nonstructural protein 1 (NS1) were predicted using three B cell epitope prediction tools, Ellipro, Bepipred and SVMTrip. Fifty sequences from each dengue serotype, representing a wide geographic area and a time span, were aligned using MEGA 6 software. The predictions were evaluated by comparing the results among the three tools and with currently available data on assay positive immunogenic epitopes of dengue NS1. A total of 22 regions on the NS1 protein ranging 6 to 21 amino acids in size were predicted as epitopes with a high probability score by the three prediction tools. Many were found to be predicted as epitopes by more than one tool, showing a good agreement in the predictions by the three tools. Further, many of the epitopes overlapped with, or partially constituted regions on the NS1 protein, which have been previously identified to be immunogenic as measured by various biochemical assays. Each of the 22 epitopes and the whole NS1 protein were then characterised for their percentages of conservation and phylogenetic relationship among and within each dengue serotypes, using IEDB and MEGA 6 analysis tools. Three epitopes were found to be highly conserved within the serotype but highly variable among the 04 dengue serotypes, suggesting that they could be used as possible serotype specific diagnostic markers. Three other epitopes were specific for the dengue group and more than $85 \%$ conserved among the four dengue serotypes, showing a potential use as dengue group specific diagnostic markers. The bioinformatics approach appears a promising method in the identification of B cell epitopes of dengue NS1 with therapeutic potential.
\end{abstract}

Keywords: B cell epitopes, bioinformatics, dengue, NS1 protein, phylogenetics.

\section{INTRODUCTION}

Dengue is one of the major public health concerns worldwide. Over 2.5 billion people, almost over $40 \%$ of the world's population are at risk from dengue. The World Health Organisation (WHO) currently estimates that there may be $50-100$ million dengue infections worldwide every year. The number of cases is increasing and the disease is also spreading to new areas (Guzman et al., 2010). Developing a vaccine against dengue has been challenging, one reason being due to cross immunity to other dengue serotypes leading to enhancement of the disease upon subsequent infections (Thisyakorn \& Thisyakorn, 2014). Recently, the first dengue vaccine Dengvaxia, a live attenuated tetravalent chimeric vaccine, developed by Sanofi Pasteur (Yauch \& Shresta, 2014), USA, was registered for use in endemic areas. After assessment for its safety, the WHO Strategic Advisory Group of Experts on Immunisation has recommended its vaccination in countries with high endemicity at national or subnational level. Its recommendation for immunisation is currently under assessment by the WHO (http://www.who.int/immunization/research/development/ dengue_vaccines/en/).

The dengue virus has a genome of approximately 11,000 bases that encodes a single large polyprotein, which is subsequently cleaved into several structural and non-structural mature peptides. The polyprotein 
is divided into three structural proteins, capsid, premembrane and envelope; seven non-structural proteins, NS1, NS2a, NS2b, NS3, NS4a, NS4b, NS5; and short non-coding regions on both 5' and 3' ends (Muller \& Young, 2013). Despite being a non-structural protein, NS1 has been observed to elicit strong humoral immune responses in mammalian cells (Muller \& Young, 2013). It is a glycoprotein of 354 amino acids. In the host cell, the NS1 protein is expressed in three forms: an endoplasmic reticulum (ER)-resident form, a membrane-anchored form, and a secreted form (sNS1) (Winkler et al., 1989; Welsch et al., 2009), the latter being responsible for this observed anti-NS1 antibody production.

The prediction of protein surface regions that are preferentially recognised by antibodies-antigenic epitopes, can help better understanding of dengue pathogenicity and development of new vaccination strategies. Moreover, diagnostic tools based on enzymelinked immunosorbent assay (ELISA) and rapid immunochromatographic assay that target NS1 have shown that high concentrations of NS1 antigens can be detected in patients with primary and secondary dengue infections at early stages of the illness (Blacksell et al., 2008). Therefore, many studies are investigating the sensitivity and specificity of sNS1 detection as a diagnostic marker during the acute phase of a dengue infection (Moi et al., 2013; Hunsperger et al., 2014; Badvay et al., 2015; Peeling et al., 2015). In a study using immunofluorescence (Youn et al., 2010) it was further observed that some NS1 epitopes could induce auto-antibodies, which then cross-react to platelets and components of the extracellular receptors inducing damage to epithelial cells. Therefore, sNS1 has been proposed to have a contributory role in the endothelial cell dysfunction that causes vascular leaking and haemorrhaging in the patients with more extreme cases of dengue.

In this study, a comprehensive analysis of B cell epitopes of dengue NS1 protein was performed using a bioinformatics approach. The prediction of $\mathrm{B}$ cell epitopes of proteins using immunocomputational programmes is useful as a first line of epitope screening, owing to their simplicity, low cost and speed. Three widely accepted epitope prediction tools were used to predict B cell epitopes on dengue NS1 protein, namely, Ellipro, Bepipred and SVMTrip. A conservational and phylogenetic analysis across and within the four dengue serotypes was performed for each of the predicted epitopes and the whole NS1 protein, in order to characterise the epitopes for their significance and potential use in dengue therapeutics and diagnostics.

\section{METHODOLOGY}

\section{Selection of NS1 sequences}

Polyprotein sequences of the four dengue serotypes (DENV1, DENV2, DENV3 and DENV4) were obtained from the National Center for Bioinfomatics (NCBI) gene bank. NS1 protein sequences (354 amino acids) were identified from the whole protein sequences and aligned using the ClustalW algorithm of MEGA 6 software. Fifty sequences from each DENV serotype were selected, which were representative of a wide geographical origin (countries from South Asia, East Asia, America and Africa, where dengue is prevalent; Table 1), and depending on the availability of the sequences these isolates spanned a time period of $10-20$ years back from 2014. Isolates with partial sequences were excluded.

Table 1: Geographical location of dengue virus of the NS1 protein sequences used in the study

\begin{tabular}{ccccc}
\hline Dengue type & South Asia & East Asia & America & Africa \\
\hline Dengue type 1 & 10 & 20 & 10 & 10 \\
Dengue type 2 & 11 & 14 & 15 & 10 \\
Dengue type 3 & 07 & 13 & 12 & 18 \\
Dengue type 4 & 04 & 12 & 24 & 10 \\
\hline
\end{tabular}

\section{Prediction of epitopes}

Three distinct tools to predict B cell epitopes utilising different epitope characteristics were used. Ellipro analysis tool and Bepipred analysis tool are available at immune epitope data base (IEDB) analysis resource (http://www.iedb.org/). Ellipro epitope prediction tool is based on the 3D structure of a protein. It implements a previously developed method that represents the protein structure as an ellipsoid, and calculates protrusion indexes for protein residues outside of the ellipsoid (Ponomarenko et al., 2008). Bepipred predicts the location of B cell epitopes using a combination of a hidden Markov model and a propensity scale method. It is mainly targeted on the properties of the epitopes, such as hydrophilicity, flexibility, accessibility, turns, exposed surface, polarity and antigenic propensity (Larsen et al., 2006; Ponomarenko et al., 2008). The third analysis tool SVMTrip, predicts epitopes using a representative sequence from a given protein. In this method, support vector machine (SVM) has been utilised by combining the tri-peptide similarity and propensity scores (SVMTrip) in order to achieve the better prediction performance (Yao et al., 2012). 


\section{Conservation and phylogenetic analysis}

Conservation of each predicted epitope and the whole NS1 sequence was analysed by using the WebLogo analysis tool (Crooks et al., 2004) and the conservation analysis tool available at IEDB. The phylogenetic trees of the whole NS1 sequence and the predicted epitope were generated using the MEGA 6 programme (Tamura et al., 2013).

\section{RESULTS}

\section{Predicted epitopes}

A total of 22 regions on the NS1 protein ranging from 6 to 21 amino acids in size were predicted as epitopes with a high probability score by the three prediction tools (Table 2). Epitopes, which were shorter than five amino acids in length were excluded. Out of the 22, eleven epitopes were predicted by Ellipro, seven from Bepiprep and four from SVMTrip (Table 2). Basically the same positions were identified as epitopes across the four serotypes, differing only one or two amino acids in length. Epitopes predicted by different tools appeared to be overlapping regions, at least partially, in many cases. For example EP5 and EP6; EP7 and EP8; EP11 and EP12; EP17 and EP18; EP18 and EP19; and EP21 and EP22 predicted by Bepiprep and Ellipro are overlapping. Moreover, EP15 and EP16 predicted by SVMTrip and Bepiprep, EP13 and EP14, and EP19 and EP20 predicted by Ellipro and SVMTrip are also overlapping epitopes. The results indicate a good agreement between the three prediction tools, where approximately the same regions have been predicted as epitopes by more than one prediction tool.

\section{Characterisation of the epitopes}

Table 2 further shows the conservation of each predicted epitope within each four serotypes as well as among the four serotypes. Most epitopes were highly conserved (more than $80 \%$ ) within a given serotype. The minimum conservation level of epitopes among the four serotypes varied from $38 \%$ to $85 \%$. Except for EP4 and EP6, all

Table 2: Dengue NS1 epitopes predicted by different B cell epitope prediction tools

\begin{tabular}{|c|c|c|c|c|c|c|c|c|}
\hline \multirow{2}{*}{$\begin{array}{l}\text { Epitope } \\
\text { ID }\end{array}$} & \multirow[t]{2}{*}{ Sequence } & \multirow[t]{2}{*}{ Size } & \multirow{2}{*}{$\begin{array}{l}\text { Predicted } \\
\text { tool / score }\end{array}$} & \multicolumn{5}{|c|}{ Conservation \% } \\
\hline & & & & All 4D & $\mathrm{D}_{1}$ & $\mathrm{D}_{2}$ & $\mathrm{D}_{3}$ & $\mathrm{D}_{4}$ \\
\hline EP1 & ${ }^{1}$ SGCVINWKGRELKCGSGIFV ${ }^{20}$ & 20 & Ellipro / 0.851 & 65 & 100 & 95 & 95 & 85 \\
\hline EP2 & ${ }^{32} \mathrm{KFQADSPKRL}^{41}$ & 10 & Bebipred & 64 & 90 & 91 & 100 & 90 \\
\hline EP3 & ${ }^{46} \mathrm{GKAWEEGV}^{53}$ & 08 & Ellipro / 0.673 & 65 & 100 & 87 & 100 & 90 \\
\hline EP4 & ${ }^{72}$ NELNHILLENDMKFT ${ }^{86}$ & 15 & Ellipro / 0.741 & 38 & 93 & 93 & 100 & 94 \\
\hline EP5 & ${ }^{102}$ MIRPQPMEYKY ${ }^{112}$ & 11 & Bebipred & 53 & 82 & 75 & 90 & 90 \\
\hline EP6 & ${ }^{103} I R P Q P M E H K Y S W K S W G K A K I I G A D T Q N T T{ }^{131}$ & 20 & Ellipro / 0.770 & 46 & 92 & 62 & 96 & 92 \\
\hline EP7 & ${ }^{134}$ IDGPNTPECPDDQRA ${ }^{148}$ & 15 & Bebipred & 63 & 86 & 86 & 93 & 92 \\
\hline EP8 & ${ }^{138} \mathrm{NTPECPDDQR}^{147}$ & 10 & Ellipro / 0.502 & 50 & 70 & 90 & 83 & 100 \\
\hline EP9 & ${ }^{155}$ EDYGFGIFT $^{163}$ & 09 & Ellipro / 0.694 & 85 & 100 & 90 & 100 & 87 \\
\hline EP10 & ${ }^{181}$ RLMSAAIKDSKAVHADMGYW ${ }^{200}$ & 20 & $\mathrm{SVM} / 0.881$ & 75 & 100 & 85 & 95 & 95 \\
\hline EP11 & ${ }^{202}$ ESEKNET $^{208}$ & 07 & Bebipred & 56 & 100 & 100 & 100 & 100 \\
\hline EP12 & ${ }^{205} \mathrm{KNETWK}^{210}$ & 06 & Ellipro / 0.664 & 55 & 100 & 90 & 100 & 100 \\
\hline EP13 & ${ }^{229}$ TLWSNGVLESEMIIPKIYGG ${ }^{248}$ & 20 & $\mathrm{SVM} / 0.286$ & 65 & 95 & 95 & 95 & 95 \\
\hline EP14 & ${ }^{230}{ }^{2} W S N G V L E S E E^{239}$ & 10 & Ellipro / 0.621 & 85 & 100 & 85 & 100 & 91 \\
\hline EP15 & ${ }^{247}$ GGPISQHNYRPGYFTQTAGPW ${ }^{267}$ & 21 & Bebipred & 72 & 95 & 90 & 92 & 90 \\
\hline EP16 & ${ }^{261}$ TQTAGPWHLGKLELDFDLCE ${ }^{280}$ & 20 & $\mathrm{SVM} / 0.704$ & 75 & 95 & 80 & 95 & 85 \\
\hline EP17 & ${ }^{277}$ DLCEGTTVVVDEHCGNRG ${ }^{294}$ & 18 & Ellipro / 0.721 & 50 & 94 & 89 & 94 & 88 \\
\hline EP18 & ${ }^{289} \mathrm{HCGNRGPSLRTTT}{ }^{301}$ & 13 & Bebipred & 63 & 92 & 93 & 92 & 87 \\
\hline EP19 & ${ }^{297}$ LRTTTVTGKIIHEWCCRSCTLP ${ }^{318}$ & 22 & Ellipro / 0.729 & 65 & 95 & 100 & 100 & 100 \\
\hline EP20 & ${ }^{310}{ }^{2}$ CCRSCTLPPLRFRGEDGCW ${ }^{329}$ & 20 & $\mathrm{SVM} / 1.000$ & 85 & 95 & 95 & 95 & 95 \\
\hline EP21 & ${ }^{335}$ RPVKEKEEN $^{343}$ & 09 & Bebipred & 64 & 100 & 85 & 87 & 89 \\
\hline EP22 & ${ }^{335}$ RPVKEKEENLVKSMVSAGSG ${ }^{354}$ & 20 & Ellipro / 0.708 & 55 & 95 & 95 & 92 & 92 \\
\hline
\end{tabular}

The given epitope sequences correspond to NS1 protein from D1 (AF298807). The measured epitope conservation ranged from a minimum percentage up to a maximum. The indicated percentages are the minimum conservations. DENV serotypes, $\mathrm{D}_{1}-\mathrm{DENV}_{1} ; \mathrm{D}_{2}-\mathrm{DENV}_{2} ; \mathrm{D}_{3}$ DENV3; $\mathrm{D}_{4}$ - DENV4. 
the other epitopes showed a conservation level of more than $50 \%$ among the four serotypes. Six epitopes were more than $70 \%$ conserved among the four serotypes with three of them, namely EP9, EP14 and EP20, having a conservation level of $85 \%$.

It was also an interesting observation that the epitope EP4 appeared highly variable among the four serotypes (only $38 \%$ conserved) but highly conserved (more than $90 \%$ ) within each serotype.

\section{Comparison of predicted epitopes with previous studies}

The epitopes predicted by the three web tools were compared with previously published epitopes, which have been shown to elicit humoral immunogenic responses as measured by various biochemical tests. A comparison of the predicted epitopes that overlap between the three tools and with previously identified antigenic regions in other studies is illustrated in Table 3. Several of the epitopes overlapped with, or partially constituted regions that have been shown to be recognised by natural monoclonal antibodies or mice antisera generated against NS1 proteins of different serotypes. Therefore, it suggests that at least parts of the predicted epitopes would be naturally immunogenic.

\section{Conservation analysis of the whole NS1 sequences}

The levels of conservation between the whole NS1 protein sequences from the four dengue serotypes and two other flavivirus groups, West Nile Virus (WNV) and Japanese Encephalitic Virus (JEV) were compared pair wise (Table 4). The conservation levels between a given DENV serotype and JEV or WNV were approximately $50 \%$ (Table 4). However, JEV and WNV showed a conservation level of more than $75 \%$ between each other. Interestingly, the carboxy terminal region of NS1 protein (271-352aa) appeared more sequence conserved across the three flavivirus groups, showing a conservation level of more than $70 \%$ (data not shown). This region has been suggested to produce cross-reactive antibodies with vascular epithelium, and has been proposed to be

Table 3: Peptide locations of dengue NS1 protein overlapping between the epitopes, predicted by different tools in the current study and in previous studies

\begin{tabular}{|c|c|c|c|}
\hline Ellipro & Bepipred & SVMTrip & Previous studies \\
\hline 103-131 (ЕP6) & 102-112 (EP5) & - & $111-130^{(\mathrm{a})} 112-123^{\text {(b) }}, 90-109^{(\mathrm{a}, \mathrm{c})}$ \\
\hline 138-147 (EP8) & $134-148$ (EP7) & - & $133-152^{(a)}$ \\
\hline \multirow[t]{2}{*}{ 155-163 (ЕР9) } & & & $154-161$ (d) \\
\hline & & 181-200 (EP10) & $187-206^{(\mathrm{a}, \mathrm{c})}$ \\
\hline 205-210 (EP12) & 202-208 (EP11) & & $193-204^{(b)}$ \\
\hline \multirow[t]{2}{*}{ 230-239 (EP14) } & & 229-248 (EP13) & \\
\hline & 247-267 (EP15) & 261-280 (EP16) & $266-274^{(b)}$ \\
\hline 277-294 (EP17) & 289-301 (EP18) & & $294-302^{(b)}$ \\
\hline 297-318 (EP19) & & 310-329 (EP20) & \\
\hline 335-354 (ЕР22) & 335-343 (EP21) & & \\
\hline
\end{tabular}

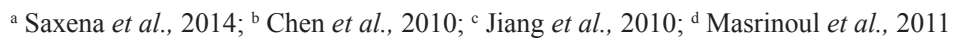

contributing to vascular leakage associated with severe dengue disease (Chen et al., 2009).

Further, dengue serotypes 1 and 3 seemed to have a closer genetic relationship with each other, as compared to the other two serotypes showing a $80 \%$ conservation level between each other. Dengue type 3 was more conserved $(98 \%)$ within the serotype than the other three serotypes (less than $92 \%$ ). The lowest conservation level within a serotype was observed with DENV4 (level $92 \%$ ).
Table 4: Conservation analysis between dengue serotype pairs and other flaviviruses

\begin{tabular}{lcccccc}
\hline $\begin{array}{l}\text { Dengue } \\
\text { type }\end{array}$ & D1 & D2 & D3 & D4 & JEV & WNV \\
\hline D1 & 96 & 73 & 80 & 68 & 51 & 49 \\
D2 & 73 & 93 & 74 & 71.5 & 53 & 55 \\
D3 & 80 & 74 & 98 & 73 & 52 & 52 \\
D4 & 68 & 71.5 & 73 & 92 & 51 & 53 \\
\hline
\end{tabular}




\section{Phylogenetic analysis of whole NS1 and the predicted epitopes}

Phylogenetic analyses were performed for whole NS1 sequences as well as for each predicted epitope from the four dengue serotypes.

The phylogenetic tree generated with whole NS1 sequences also showed that the serotypes 1 and 3 were more closely related (Figure 1). Serotype 2 has branched off before serotypes 1 and 3 have separated. Serotype 4 was the most distant from the other serotypes, and appears as an isolated group in the phylogenetic tree. Further, dengue strains with different geographical origins have clustered into 4 distinctly separated groups, an observation which was evident with each serotype. This indicates a geographical segregation of DENV strains into four main locations, namely, America, South Asia, Africa and South East Asia.

Sequence heterogeneity among the strains from a given serotype shows additional levels of organisation into subtypes within a given serotype. This can be evidenced in the phylogenetic tree. The strains of dengue serotype 3 organised into a fewer number of subgroups within the serotype, showing a higher level of conservation within the particular serotype. In contrary, serotype 4 showed a higher number of subgroups, reflecting a low level of conservation within serotype 4 .

The phylogenetic trees generated for each predicted epitope generally followed the same pattern of phylogenetic relationship observed for the whole NS1 sequences, except for a few epitopes as described below. In EP2, serotypes 1 and 3 sequences were seen to group into a single group rather than into two separate groups, indicating that epitopes 1 and 3 are highly similar across the two serotypes. Interestingly, in the three epitopes EP4, EP11 and EP12, all the four serotypes were well separated being more or less equally distant from each other (Figure 2). The result is compatible with the results of conservation analysis of the same epitopes, where they were highly variable among the four serotypes while being highly conserved within a given serotype. Further, in agreement with the high conservation level among DENV serotypes observed for EP9, all four dengue serotypes appeared as one group rather than as four groups in the phylogenetic tree generated for EP9 epitope (Figure 3).

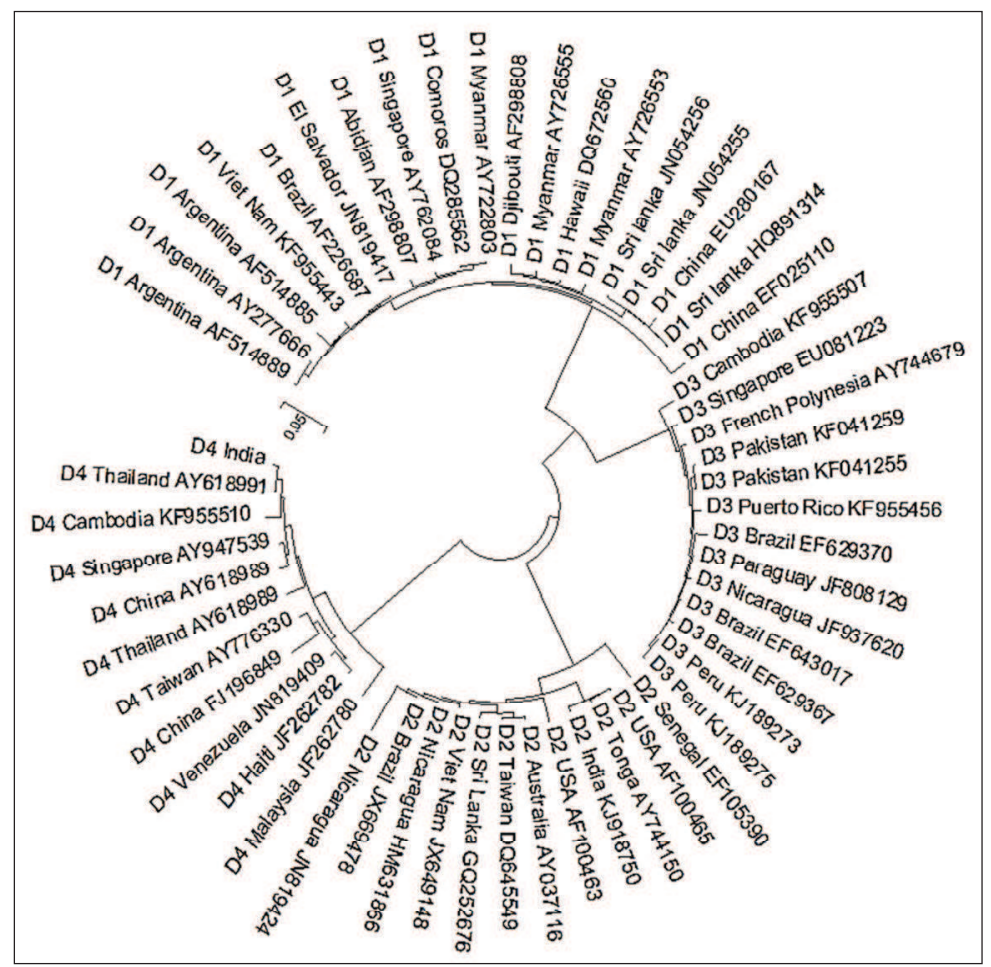

Figure 1: Phylogenetic tree generated for whole NS1 sequence

Note: serotype 2 branches off before serotypes 1 and 3 separate out. Serotype 4 is the most distant of the four. Only $10-15$ sequences from each serotype are shown in the figure for readability. 


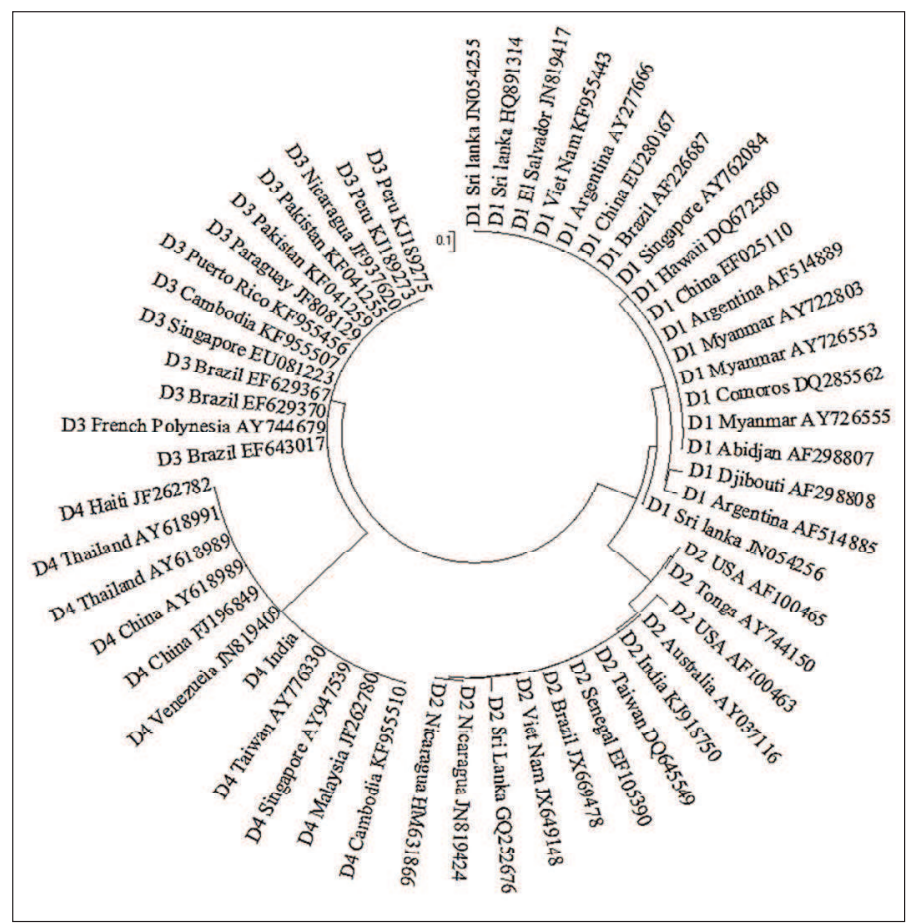

Figure 2: Phylogenetic tree generated for EP4

Note: all four serotypes are separated from each other to form isolated individual clusters with a fewer sub groups, indicating high diversity between the four serotypes and high conservation within a serotype. Only $10-15$ sequences from each serotype are shown in the figure for readability.

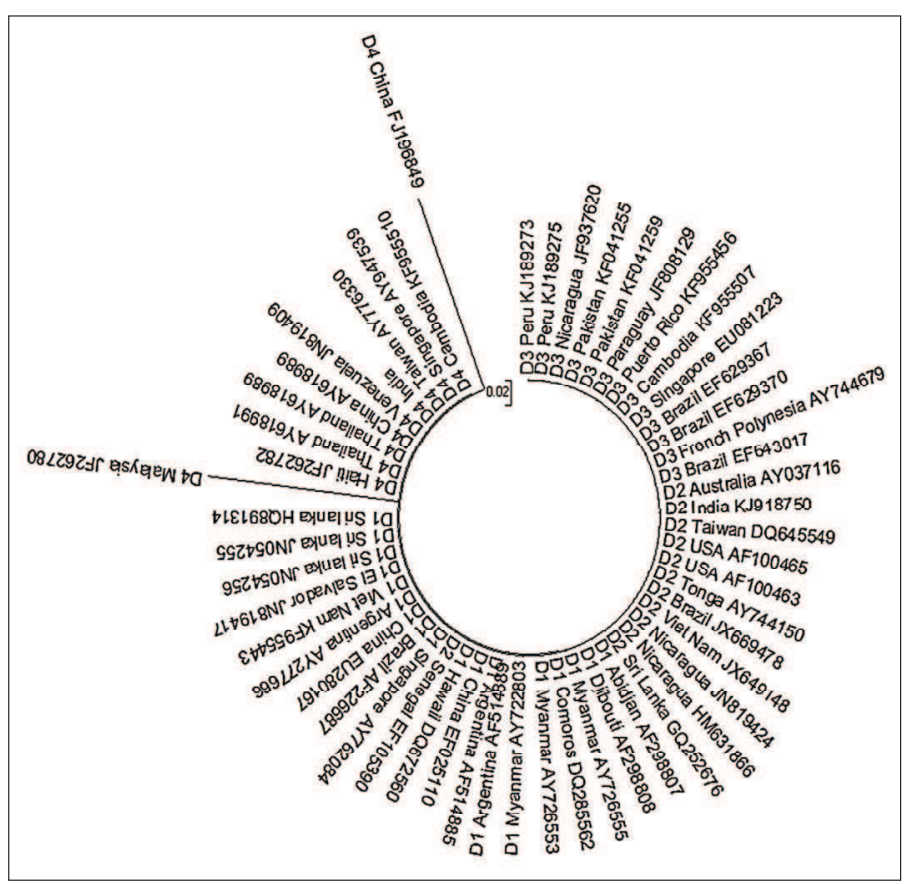

Figure 3: Phylogenetic tree generated for EP9

Note: less segregation between the four serotypes, and rather appears as one large group indicating less diversity across the serotypes. Only $10-15$ sequences from each serotype are shown in the figure for readability. 


\section{DISCUSSION}

The present study is an attempt to identify linear B cell epitopes of NS1 protein as predicted by immunocomputational tools and to characterise those epitopes bioinformatically, in order to understand their significance and use in dengue disease diagnosis and therapeutics. These immunocomputational tools would be especially useful as a first line of screening in the identification of potential dengue protein antigens owing to their low cost and resources, which can be followed by a validation with laboratory experiments. Therefore in the present study, we compared the epitope predictions between the different tools as well as with the comparable studies where the immunogenicity of the epitopes have been biochemically tested and proven. We further conducted conservation analysis of all the predicted epitopes as well as phylogenetic analysis of the potentially significant predicted epitopes, in comparison with the whole NS1 protein sequences.

Three distinct epitope prediction tools were used to identify the epitopes of dengue NS1 protein. The three tools utilise three different approaches and epitope characteristics into their predictions. It was a promising result that some of the epitope regions were predicted by more than one prediction tool. In line with this speculation many of these epitopes have already been identified to induce humoral immune responses as well according to previous studies (Table 4). The good agreement of the results between the three tools and the previous studies might indicate high chances for those predicted epitopes to represent true antigens under natural circumstances. Therefore, epitope prediction using immunocomputational methods appears a reliable method to screen B cell epitopes owing to its simplicity and the use of less resources.

A few of these predicted epitopes were further suggestive to be important in terms of their potential use as a diagnostic marker or vaccine candidate, as described below.

In the characterisation of the predicted epitopes, some of them (6 out of 22) were found to be conserved more than $70 \%$ among the four serotypes. Conserved epitopes are advantageous in the preparation of subunit vaccines as they would be broadly effective for all serotypes and strains. Out of these six, epitope EP9 (constituting the region 155-163), epitope E14 (constituting the region 230-239), and EP20 (constituting the region 310-329) are highly conserved (more than $85 \%$ ). These epitopes from DENV further have less than $25 \%$ conservation with the corresponding epitope sequences from JEV or WNV. Therefore, the three epitopes would be good candidates to be developed into group detection diagnostic markers for dengue. Further, a previous study conducted to screen conserved epitopes on dengue NS1 protein using cross reactive monoclonal antibodies has identified the region 141-163 as an immunodominant common epitope for all four serotypes (Masrinoul et al., 2011). EP9 predicted in the current study falls within this region. EP9 therefore can be considered as a potential candidate for designing a broadly reactive subunit vaccine against NS1 or for developing into a diagnostic tool for group detection. EP14 also appears to have such potential given that its immunodominancy is proven using biochemical tests. EP20 on the other hand, is located in the C-terminal region of the protein, antibodies against which has been suggested to cross-react with the vascular endothelium. Since this has been proposed as a possible contributing factor for vascular leakage associated with severe dengue (Chen et al., 2009), the potential of EP20 as a vaccine target will be limited.

EP4 is another interesting epitope. According to the conservation analysis results for the predicted epitopes, EP4 demonstrated a high potentiality to be used as a serotype specific diagnostic marker. Within each serotype its conservation was very high. However, among the four serotypes, the overall conservation was very low $(38 \%)$. The results indicate that the epitope sequence is very variable among the four serotypes, but highly similar within a given serotype. The observation was also evident in the phylogenetic tree generated for EP4, where each serotype branched off into well isolated equally distant groups. Further, Chen et al. (2010) has identified the NS1 protein range $72-85$ from DENV1 as an immunodominant epitope, which is serotype specific but conserved within that serotype. This region coincides well with the predicted epitope EP4, which runs from 72-86. Therefore, our observations are in good agreement with the findings of Chen et al. (2009) demonstrating that EP4 would be an ideal candidate to be developed into a diagnostic tool for specific identification of the four dengue serotypes.

In line with the results from conservation analysis of whole NS1 squence, the genetic diversity among the strains from serotype 4 appeared higher than what was observed within the other three serotypes. Not only that, dengue type 4 was phylogenetically the most deviant from other three serotypes. Further, as evidenced also in Wang et al. (2000), dengue types 1 and 3 appeared phylogenetically the closest pair among the four serotypes showing a more than $80 \%$ conservation between each 
other. Collectively, the dengue group shared only a conservation level of $50 \%$ with the NS1 protein from two other flavivirus groups, JEV or WNV. However, it was interesting to note the carboxy terminal region (271$352 \mathrm{aa}$ ) of the protein, which has been suggested to be pathologically important (Chen et al., 2009) being more conserved across different flavivirus groups. Further understanding of the genetic variability or similarity among and within the serotypes of dengue virus as well as across different flavivirus groups would provide insight into the understanding of dengue pathogenesis.

Finally, we observed geographical segregation of dengue strains with each serotype into 4 main regions as South Asia, East Asia, Africa and America. This type of geographical segregation has also been reported in Murray et al. (2013). In this line, it would also be interesting and suggestive as a future study to see the dominancy in different serotypes in each region across the time line, which could be indicative of future outbreaks of DENV serotypes in different locations.

\section{CONCLUSION}

The linear B cell epitopes of NS1 predicted by the three immunocomputational tools, Ellipro, Bebiprep and SVMTrip, are in good agreement with each other as well as with immunodominant epitopes identified in previous studies. The predicted epitopes, EP9 and EP14, have the potential as vaccine targets or diagnostic markers for dengue group detection. The epitope EP4 appears as a good marker for an antibody based serotype detection diagnostic tool for dengue. These epitopes have to be further validated using laboratory tests before their usage in potential applications. The bioinformatics approach used in the current study appears promising as an initial epitope screening technique to minimise the cost and time in the identification of $\mathrm{B}$ cell epitopes of dengue NS1 with therapeutic potential.

\section{Acknowledgement}

The authors thank the General Sir John Kotelawala Defence University for providing the necessary resources to undertake the research. Further, Prof. Aravinda de Silva, University of North Carolina for his guidance, and the National Science Foundation, Sri Lanka that facilitated the establishment of the said collaboration (IPSAT/2014/1 grant) are gratefully acknowledged.

\section{REFERENCES}

1. Badave G.K., Swaroop P.S. \& Rao P.N. (2015). Importance of NS1 antigen detection and its association with platelet count for early diagnosis of dengue virus infection. International Journal of Current Microbiology and Applied Science 4(3): $779-784$.

2. Blacksell S.D., Mammen M.P., Thongpaseuth S., Gibbons R.V., Jarman R.G., Jenjaroen K., Nisalak A., Phetsouvanh R., Newton P.N. \& Day N.P. (2008). Evaluation of the Panbio dengue virus nonstructural 1 antigen detection and immunoglobulin $\mathrm{M}$ antibody enzyme-linked immunosorbent assays for the diagnosis of acute dengue infections in Laos. Diagnostic Microbiology and Infectious Disease 60(1): $43-49$.

DOI: http://dx.doi.org/10.1016/j.diagmicrobio.2007.07.011

3. Chen M.C., Lin C.F., Lei H.Y., Lin S.C., Liu H.S., Yeh T.M., Anderson R. \& Lin Y.S. (2009). Deletion of the $\mathrm{C}$-terminal region of dengue virus nonstructural protein 1 (NS1) abolishes anti-NS1-mediated platelet dysfunction and bleeding tendency. The Journal of Immunology 183(3): $1797-1803$.

DOI: http://dx.doi.org/10.4049/jimmunol.0800672

4. Chen Y., Pan Y., Guo Y., Qiu L., Ding X. \& Che X. (2010). Comprehensive mapping of immunodominant and conserved serotype- and group-specific B-cell epitopes of nonstructural protein 1 from dengue virus type 1. Virology 398(2): $290-298$.

DOI: http://dx.doi.org/10.1016/j.virol.2009.12.010

5. Crooks G.E., Hon G., Chandonia J.M. \& Brenner S.E. (2004). WebLogo: a sequence logo generator. Genome Research 14(6): $1188-1190$.

DOI: http://dx.doi.org/10.1101/gr.849004

6. Guzman M.G. et al., (15 authors) (2010). Dengue: a continuing global threat. Nature Reviews Microbiology 8(12 Suppl): S7 - 16 .

DOI: http://dx.doi.org/10.1038/nrmicro2460

7. Hadinegoro S.R. et al., (24 authors) (2015). Efficacy and long-term safety of a dengue vaccine in regions of endemic disease. The New England Journal of Medicine 373: 1195 $-1206$.

DOI: http://dx.doi.org/10.1056/NEJMoa1506223

8. Hunsperger E.A. et al., (18 authors) (2014). Evaluation of commercially available diagnostic tests for the detection of dengue virus NS1 antigen and anti-dengue virus IgM antibody. Plos Neglected Tropical Diseases 8(10): e3171. DOI: http://dx.doi.org/10.1371/journal.pntd.0003171

9. Jiang L., Zhou J.M., Yin Y., Fang D.Y., Tang Y.X. \& Jiang L.F. (2010). Selection and identification of B-cell epitope on NS1 protein of dengue virus type 2. Virus Research 150 $(1-2): 49-55$.

10. Larsen J.E., Lund O. \& Nielsen M. (2006). Improved method for predicting linear B-cell epitopes. Immunome Research 2: $2-10$. 
DOI: http://dx.doi.org/10.1186/1745-7580-2-2

11. Masrinoul P., Diata M.O., Pambudi S., Limkittikul K., Ikuta K. \& Kurosu T. (2011). Highly conserved region 141168 of the NS1 protein is a new common epitope. Japanese Journal of Infectious Diseases 64(2): 109 - 115.

12. Moi M.L. et al., (11 authors) (2013). Detection of dengue virus nonstructural protein 1 (NS1) by using ELISA as a useful laboratory diagnostic method for dengue virus infection of international travellers. Journal of Travel Medicine 20(3): 185 - 193.

DOI: http://dx.doi.org/10.1111/jtm.12018

13. Muller D.A. \& Young P.R. (2013). The flavivirus NS1 protein: molecular and structural biology, immunology, role in pathogenesis and application as a diagnostic biomarker. Antiviral Research 98(2): 192 - 208.

DOI: http://dx.doi.org/10.1016/j.antiviral.2013.03.008

14. Murray N.E., Quam M.B. \& Wilder-Smith A. (2013). Epidemiology of dengue: past, present and future prospects. Clinical Epidemiology 5: 299 - 309.

15. Peeling R.W. et al., (19 authors) (2010). Evaluation of diagnostic tests: dengue. Nature Reviews Microbiology 8(12): $530-538$.

DOI: http://dx.doi.org/10.1038/nrmicro2459

16. Ponomarenko J., Bui H.H., Li W., Fusseder N., Bourne P.E., Sette A. \& Peters B. (2008). ElliPro: a new structurebased tool for the prediction of antibody epitopes. BMC Bioinformatics 9: $514-518$.

DOI: http://dx.doi.org/10.1186/1471-2105-9-514

17. Saxena N., Pandey A., Ahuja A.J. \& Kumar V. (2014). Integration of in-silico approaches for designing B cell epitopes for disease diagnosis. International Journal of Current Research 131(2): 153 - 168.

18. Tamura K., Stecher G., Peterson D., Filipski A. \& Kumar S. (2013). MEGA6: molecular evolutionary genetics analysis version 6.0. Molecular Biology and Evolution
30(12): $2725-2729$.

DOI: http://dx.doi.org/10.1093/molbev/mst197

19. Thisyakorn U. \& Thisyakorn C. (2014). Latest developments and future directions in dengue vaccines. Therapeutic Advances in Vaccines 2(1): 3 - 9.

DOI: http://dx.doi.org/10.1177/2051013613507862

20. Wang E., Ni H., Xu R., Barrett A.D., Watowich S.J., Gubler D.J. \& Weaver S.C. (2000). Evolutionary relationships of endemic/epidemic and sylvatic dengue viruses. Journal of Virology 74: 3227 - 3234 .

DOI: http://dx.doi.org/10.1128/JVI.74.7.3227-3234.2000

21. Welsch S., Miller S., Romero-Brey I., Merz A., Bleck C.K., Walther P., Fuller S.D., Antony C., Krijnse-Locker J. \& Bartenschlager R. (2009). Composition and threedimensional architecture of the dengue virus replication and assembly sites. Cell Host and Microbe 5(4): 365 - 375. DOI: http://dx.doi.org/10.1016/j.chom.2009.03.007

22. Winkler G., Maxwell S.E., Ruemmler C. \& Stollar V. (1989). Newly synthesized dengue-2 virus nonstructural protein NS1 is a soluble protein but becomes partially hydrophobic and membrane-associated after dimerization. Virology 171(1): $302-305$.

23. Yao B., Zhang L., Liang S. \& Zhang C. (2012). A method to predict antigenic epitopes using support vector machine to integrate tri-peptide similarity and propensity. PLOS ONE 7(9): e45152.

DOI: http://dx.doi.org/10.1371/journal.pone.0045152

24. Yauch L.E. \& Shresta S. (2014). Dengue virus vaccine development. Advances in Virus Research 88: 315 - 372. DOI: http://dx.doi.org/10.1016/B978-0-12-800098-4.00007-6

25. Youn S., Cho H., Fremont D.H. \& Diamond M.S. (2010). A short terminal peptide motif on flavivirus nonstructural protein NS1 modulates cellular targeting and immune recognition. Journal of Virology 84(18): 9516 - 9532. DOI: http://dx.doi.org/10.1128/JVI.00775-10 